\title{
Tomografia computadorizada na insuficiência respiratória aguda
}

\author{
SILVIA REgINA RIOS VIEIRA ${ }^{1}$
}

O objetivo do presente trabalho é revisar o uso da tomografia computadorizada (TC) na insuficiência respiratória aguda (IRA) - lesão pulmonar aguda ou acute lung injury (ALI) e síndrome da angústia respiratória aguda (SARA). Foram revisados os principais trabalhos publicados na literatura em língua inglesa e localizados por pesquisa na Medline, que estudaram o uso da TC na IRA. A TC permite, num primeiro momento, uma avaliação qualitativa da morfologia pulmonar buscando a presença de hiperdensidades difusas e/ ou localizadas e de outras anormalidades concomitantes (como derrame pleural e pneumotórax). Além disso, permite avaliar os histogramas de densidade pulmonares a partir dos quais podem ser efetuados o cálculo dos volumes pulmonares totais e regionais (volume das regiões ventiladas, parcialmente ventiladas, não ventiladas e de hiperdistensão). Isso possibilita quantificar recrutamento alveolar e hiperdistensão decorrentes de estratégias ventilatórias, como por exemplo do uso de diferentes níveis de pressão expiratória final positiva (PEEP). (J Pneumol 1999;25(5):294-298)

\section{Computerized tomography in acute respiratory failure}

The goal of this paper is to review the use of CT scan in acute respiratory failure (ARF), acute lung injury (ALI) and acute respiratory distress syndrome (ARDS). The most important papers studying CT in ARF, published in English language literature, were found in Medline. The use of CT in patients with ARF let allowed the authors to proceed to a qualitative evaluation of lung morphology looking for diffuse an/or localized hyperdensities, and for other concomitant abnormalities (pleural effusion, pneumothorax). It is also possible to evaluate lung density histograms and calculate the gas-tissue ratio within the lungs. Likewise, one can also calculate total and regional lung volumes (aerated, poorly-aerated, non-aerated, and overdistension volumes). Knowing those volumes it is possible to quantify alveolar recruitment and overdistension due to ventilatory strategies as the use of different positive end expiratory pressure (PEEP) levels.

Descritores - Lesão pulmonar aguda. Síndrome da angústia respiratória aguda. Tomografia computadorizada. Recrutamento alveolar. H iperdistensão.

Key words - A cute lung injury. A cute respiratory distress syndrome. Computerized tomography. Alveolar recruitment. Overdistension.

A tomografia computadorizada (TC) tem sido utilizada para avaliar pacientes com insuficiência respiratória aguda (IRA), incluindo lesão pulmonar aguda ou acute lung injury (ALI) e síndrome da angústia respiratória aguda (SARA). 0 primeiro relato do uso da TC na SARA em humanos foi publicado em

1. Professora do Centro de Tratamento Intensivo do Hospital de Clínicas de Porto A legre - Departamento de Medicina Interna, UFRGS. Doutora em Medicina pela Universidade Federal do Rio Grande do Sul.

Endereço para correspondência - Silvia Regina Rios Vieira, São Luís, 1.127, A pto. 501 - 90620-170 - Porto Alegre, RS. Tel./ fax (051) 223-4256; E-mail: srvieira@zaz.com.br

Recebido para publicação em 19/2/99. Aprovado, após revisão, em 1/3/99.
Siglas e abreviaturas utilizadas neste trabalho

IRA - Insuficiência respiratória aguda

ALI - Lesão pulmonar aguda

SARA - Síndrome da angústia respiratória aguda

PEEP - Pressão expiratória final positiva

CTN - Números de tomografia computadorizada

CRF - Capacidade residual funcional

CPT - Capacidade pulmonar total

ZEEP - Zero de pressão expiratória final positiva

$1983^{(1)}$. Desde então a TC tem sido utilizada para estudar os aspectos morfológicos e fisiopatológicos da síndrome e para avaliar os efeitos de algumas modalidades terapêuticas empregadas em seu manejo(2-12).

0 uso da TC na SARA permite, num primeiro momento, uma análise qualitativa que, quando realizada por profissional experiente, possibilita localizar com precisão hiperdensidades predominantemente difusas ou localizadas e identificar a presença de outras alterações associadas entre as quais derrame pleural, empiema, abscesso pulmonar e pneumotó$\operatorname{rax}^{(13)}$. As hiperdensidades pulmonares identificadas por TC 
ocorrem predominantemente nas regiões do pulmão dependentes de uma maior pressão hidrostática, sendo, portanto, mais freqüentes nas regiões posteriores quando os pacientes estão em decúbito dorsal(2-4,6,7,9,10,14,15). Essa distribuição já havia também sido demonstrada em animais de experimentação com SARA induzida por ácido oléico (16,17).

O emprego de uma única seção tomográfica já permite uma avaliação bidimensional das dimensões pulmonares ao longo do eixo ântero-posterior ou transversal. A tualmente, as novas gerações de tomógrafos permitem a realização de TCs espiraladas que realizam cortes tomográficos múltiplos e contínuos, desde o ápex até a base, durante um curto período de apnéia, possibilitando uma avaliação tridimensional dos pulmões e permitindo, com isso, a medida do volume pulmonar total ao longo dos diâmetros ântero-posterior, transversal e cefalocaudal.

Duas análises diferentes podem ser usadas para a obtenção dos volumes pulmonares com base nos números de tomografia computadorizada (computed tomographic number ou CTN) fornecidos pelo tomógrafo. A primeira delas foi proposta por Gattinoni et al . ${ }^{(4,6,15)}$ e baseia-se na pressuposição de que os CTNS de um dado volume pulmonar correlacionam-se com a proporção de gás e tecido dentro do pulmão. U sando essa análise, é possível computar o volume de gás e de tecido de todo o pulmão. Resumidamente, apoia-se no conceito de que o CTN médio - ou densidade média - de um determinado volume pulmonar correlaciona-se com a respectiva proporção de gás e tecido no interior do pulmão. 0 CTN que caracteriza cada compartimento pulmonar é expresso em unidades $\mathrm{H}$ ounsfield $(\mathrm{UH})$ e é definido como o coeficiente de atenuação radiológico do material em estudo menos o coeficiente de atenuação da água dividido pelo coeficiente de atenuação da água. O CTN da água é OU H, O CTN do ar é $-1.000 \mathrm{UH}$ e oS CTNS dos diferentes cortes pulmonares são distribuídos ao longo de uma escala de $-1.000 \mathrm{UH}$ a $+100 \mathrm{UH}$. U ma região pulmonar caracterizada por um CTN médio de - 500 U H é considerada como sendo composta por $50 \%$ de gás ou ar e $50 \%$ de água ou tecido. Considerando-se que os pulmões, quer sadios quer doentes, são basicamente compostos por ar e tecido e que a escala de densidades pulmonares em unidades Hounsfield é uma escala linear entre - 1.000 U (densidade do ar) e 0 (densidade da água), podese admitir que um pulmão com um volume de 2 litros e um número de densidade médio de $-500 \mathrm{UH}$ seja composto por 1 litro de ar e 1 litro de água e/ ou tecido(6). U sando essa análise, é possível aferir o volume de gás e tecido presente nos pulmões. Num primeiro momento, calculam-se os volumes de gás e de tecido de cada compartimento pulmonar e, posteriormente, os volumes de gás e tecido de todo o pulmão.

U ma outra abordagem fundamenta-se na medida dos volumes pulmonares a partir da análise dos histogramas de densidade pulmonares ${ }^{(12)}$. A técnica baseia-se no cálculo do volume de cada píxel (unidade de área adotada em Tc) que passa a ser chamado vóxel (unidade de volume empregada em TC). Numa tomografia volumétrica helicoidal, registrada em um campo de 35 por $35 \mathrm{~cm}^{2}$, em cortes de $1 \mathrm{~cm}$ de espessura e em um nível de zum de 1 , sabe-se que cada píxel corresponde à unidade de superfície e cada vóxel à unidade de volume e que: 1) a superfície de cada seção é igual a $35 \mathrm{x}$ $35 \times 1 \mathrm{~cm}^{2}$, que corresponde a $512 \times 512$ píxeis; 2) o volume de cada seção é igual a $35 \times 35 \times 1 \mathrm{~cm}^{3}$, que corresponde a 512 × 512 vóxeis. Dessa forma, um vóxel é igual a 0,0047 $\mathrm{ml}$ quando 0 valor de zum usado para o registro tomográfico é 1 . Um fator de correção apropriado deve ser aplicado se um zum diferente é usado na aquisição das imagens. Para cada corte tomográfico o pulmão é normalmente dividido em 50 compartimentos de $22 \mathrm{UH}$ cada um. Os volumes pulmonares de cada compartimento são calculados multiplicando-se o número de píxeis pelo volume de cada píxel. A partir da adição dos volumes pulmonares dos diferentes compartimentos obtêm-se ou os volumes pulmonares totais ou os volumes pulmonares divididos conforme a região desejada. Para distinguir zonas pulmonares com diferentes graus de ventilação, os pulmões têm sido divididos, de acordo com Gattinoni(5), em três regiões: zonas aeradas localizadas entre -1.000 e - $500 \mathrm{UH}$, zonas pobremente aeradas entre -500 e $-100 \mathrm{UH}$ e zonas não aeradas entre $-100 \mathrm{e}+100 \mathrm{UH}$.

A grande vantagem da TC como ferramenta para medida dos volumes pulmonares é sua alta precisão e boa reprodutilidade. Ela é mais acurada do que outros métodos empregados para medir a capacidade residual funcional, entre os quais as que utilizam lavagem e diluição de gases, como nitrogênio, hexafluoreto sulfúrico e hélio(18-21). A TC oferece também a possibilidade de medir separadamente os volumes dos lobos inferiores e superiores, bem como de quantificar derrame pleural. A possibilidade de medir volume de diferentes compartimentos permite uma determinação acurada do recrutamento alveolar e da distensão pulmonar induzidos pela ventilação mecânica, desde que sejam aferidos os volumes das áreas correspondentes.

É importante reconhecer o limite da hiperdistensão pulmonar, para que se possa quantificar esse efeito deletério da ventilação mecânica na SARA. A té o momento, porém, não existe conhecimento exato sobre tal limite. Recentemente, nove pacientes foram submetidos a um protocolo que incluía TC com cálculo do volume pulmonar a partir dos histogramas de densidade ${ }^{(22)}$ visando estudar os efeitos da pressão expiratória final positiva ou PEEP e diferentes volumes correntes no recrutamento alveolar e na hiperinflação pulmonar. Foram utilizados três cortes tomográficos: 0 primeiro (superior) no nível do arco aórtico, o segundo (médio) no nível da carena e o terceiro (inferior) $5 \mathrm{~cm}$ abaixo do segundo. Foram considerados como hiperinflação os volumes pulmonares medidos na região -1.000 a $-800 \cup H$. Conforme os próprios autores reconheceram, essa foi uma definição 
arbitrária, porque nenhum dado definitivo foi adotado para estabelecer como limite de hiperdistensão o valor de-800U H.

Pacientes com SARA freqüentemente são submetidos a TCS com injeção de material contrastante, com vistas a estabelecer melhor diferenciação entre consolidações e derrames pleurais ${ }^{(12)}$. A pesar do uso rotineiro, ainda não está devidamente esclarecido se o material de contraste pode ou não causar alguma interferência nos histogramas de densidade pulmonares e nos cálculos dos volumes pulmonares deles derivados.

Em geral, ao se analisarem as alterações encontradas em um determinado exame e em uma dada patologia, elas costumam ser comparadas com as características encontradas em indivíduos sadios. Existem poucos estudos nos quais voluntários sadios tenham sido submetidos a TC a fim de avaliar seus histogramas de densidade pulmonares ${ }^{(4-6)}$. Nessas publicações, a Tc de tórax foi realizada em três cortes tomográficos (ápex, hilo e base) e as exposições foram tomadas ao final da expiração normal, em apnéia, ao nível da pressão atmosférica e sem injeção de material de contraste. Os histogramas de densidade pulmonares mostraram uma distribuição unimodal com um volume máximo localizado na faixa de -700 a $-800 \mathrm{UH}^{(4,5)}$ e com CTN médio de $-670 \mathrm{UH}^{(6)}$. Não houve tentativa de avaliar limite de hiperinflação em voluntários sadios.

Os principais objetivos do emprego da TC em pacientes com SARA seriam, portanto: avaliar qualitativamente a morfologia pulmonar buscando a presença de hiperdensidades difusas e/ ou localizadas e de outras anormalidades concomitantes (derrame pleural, pneumotórax); avaliar os histogramas de densidade pulmonares e, a partir deles, calcular os valores pulmonares do pulmão como um todo e das diferentes regiões de interesse; calcular os graus de recrutamento alveolar e de hiperdistensão decorrentes de diferentes intervenções ventilatórias a partir do cálculo dos distintos volumes pulmonares.

Recentemente realizamos dois trabalhos, o primeiro envolvendo 0 uso de TC em voluntários sadios ${ }^{(23)}$ e o segundo utilizando TC para avaliar efeitos da PEEP em pacientes com IRA $^{(24)}$.

No primeiro estudo(23), os objetivos foram: 1) avaliar a distribuição dos histogramas de densidade pulmonares em voluntários sadios em capacidade residual funcional (CRF) e em capacidade pulmonar total (CPT), a fim de determinar o limite de densidade para diferenciar o pulmão normalmente ventilado da hiperdistensão; 2) uma vez determinado em voluntários sadios, testar esse limite em pacientes com IRA e em ventilação mecânica, para distinguir o recrutamento PEEPinduzido da hiperdistensão PEEP-induzida; 3) avaliar a influência do material de contraste injetado durante as TCs na distribuição dos histogramas de densidade pulmonares.

Neste estudo(23), foram avaliados por TC espiralada de tórax seis voluntários sadios e seis pacientes com IRA. N os pri- meiros a tomografia foi feita, tanto antes como após injeção de $80 \mathrm{ml}$ de contraste, em CRF e em CPT contra pressão de $30 \mathrm{CmH}_{2} \mathrm{O}$, pressão essa que visava desencadear hiperdistensão. Nos pacientes com IRA a TC foi realizada em ZEEP (zero de PEEP) e em PEEP.

0 resultado mais importante deste estudo(23) foi que, em voluntários sadios, o limite de densidade separando parênquima pulmonar normalmente ventilado de hiperdistensão pulmonar foi definido como sendo de cerca de $-900 \mathrm{UH}$. Ao utilizarmos esse limite de densidade como limite de hiperdistensão nos seis pacientes com IRA, foi possível demonstrar que o recrutamento alveolar PEEP-induzido foi acompanhado por um certo grau de distensão pulmonar em pelo menos alguns pacientes. Lembramos que recrutamento alveolar corresponde à diminuição da quantidade de pulmão não ventilado (densidade entre -100 e +100 unidades H ounsfield).

A TC torácica forneceu medidas acuradas dos histogramas de densidade pulmonares e a partir dessas medidas os volumes pulmonares totais e regionais (normalmente ventilados, parcialmente ventilados, não ventilados e com hiperdistensão) puderam ser determinados, permitindo avaliar os efeitos de uma dada estratégia ventilatória, como por exemplo PEEP, na morfologia pulmonar.

Com relação aos efeitos da PEEP em pacientes com IRA, a avaliação tomográfica de nosso estudo(23) corroborou estudos prévios ${ }^{(22)}$ confirmando que recrutamento alveolar induzido pela PEEP é seguidamente acompanhado de hiperdistensão. Entretanto julgamos, que a magnitude do fenômeno foi hiperestimada por Dambrosio et al. ${ }^{(22)}$ ao considerarem como intervalo de hiperdistensão $-800 \mathrm{a}-1.000 \mathrm{UH}$ e não 0 que, conforme delimitamos, se encontrou entre -900 e $-1.000 \mathrm{UH}$. Deve também ser salientado que esse limite pode variar levemente de um centro para outro. O uso dos CTNS como valores absolutos é questionável(25), uma vez que podem ser influenciados pelo tipo de tomógrafo, pela voltagem e pelos algoritmos de reconstrução tomográfica. Idealmente, o limite de densidade da hiperdistensão pulmonar deveria ser reavaliado sempre que um novo tomógrafo fosse introduzido.

O utro resultado importante do estudo(23) foi a ausência de influência da injeção de material de contraste na distribuição dos histogramas de densidade pulmonares e no cálculo subseqüente dos diferentes volumes pulmonares. Esse resultado é importante uma vez que material de contraste é usado freqüentemente quando TCS são realizadas em pacientes com SARA, visando diferenciar parênquima pulmonar consolidado de derrame pleural.

Num segundo trabalho foi estudado o significado do ponto de inflexão inferior da curva pressão-volume em pacientes com insuficiência respiratória aguda utilizando-se avaliação por tomografia computadorizada(24). 0 objetivo desta investigação foi avaliar se a presença ou ausência de um ponto de inflexão inferior na curva pressão-volume correspondia a di- 
ferenças na morfologia pulmonar que causassem respostas diversas a distintos níveis de PEEP, em termos de recrutamento alveolar e hiperdistensão PEEP-induzidos, em pacientes com IRA.

Neste trabalho, foram estudados, consecutivamente, 14 pacientes com o diagnóstico de IRA - ALI OU SARA. Nestes pacientes medidas respiratórias e hemodinâmicas, curvas pressão-volume e TC espiralada de tórax foram realizadas em ZEEP e em do is níveis de PEEP. 0 primeiro nível de PEEP (PEEP1) foi definido como igual ao ponto de inflexão inferior da curva pressão-volume +2 ou igual a 10 na ausência desse ponto. 0 segundo nível de PEEP (PEEP2) foi definido como igual ao ponto de inflexão inferior da curva pressão-volume +7 ou igual a 15 na ausência desse ponto. Os pacientes foram divididos em dois grupos de acordo com a presença ou ausência do ponto de inflexão inferior na curva pressão-volume.

Nesta série de pacientes com IRA avaliados por TC ${ }^{(24)}$, 0 volume de pulmão caracterizado por densidade de tecido foi semelhante, sugerindo que o dano alveolar foi comparável em todos eles. Porém dois grupos de pacientes foram identificados, diferindo pela curva pressão-volume e pela morfologia pulmonar. 0 primeiro grupo foi caracterizado por curvas pressão-volume toracopulmonares e pulmonares demonstrando complacência respiratória reduzida e presença de um ponto de inflexão inferior. Nesse grupo predominavam hiperdensidades pulmonares difusas, presentes nos lobos superiores e inferiores. 0 segundo grupo apresentava complacência respiratória mais alta sem evidência de ponto de inflexão inferior nas curvas pressão-volume. Nesse grupo predominavam hiperdensidades pulmonares distribuídas nos lobos inferiores, enquanto os lobos superiores permaneciam ventilados. Em ambos os grupos, PEEP induziu um recrutamento alveolar significativo que foi acompanhado por hiperdistensão pulmonar apenas nos pacientes do grupo sem ponto de inflexão inferior, sendo esta hiperdistensão maior quanto maiores níveis de PEEP forem aplicados. É importante lembrar que todos os pacientes deste estudo que apresentaram ponto de inflexão inferior nas curvas toracopulmonares, também o apresentaram nas pulmonares, mostrando a contribuição do componente pulmonar na determinação do referido ponto. Não tivemos casos como os de Mergoni et al.(26), em que o ponto de inflexão inferior da curva toracopulmonar era relacionado apenas ao componente torácico, sem a participação do pulmão, situação em que o comportamento pode diferir do por nós observado.

A diferente resposta à PEEP entre os do is grupos de pacientes ${ }^{(24)}$ pode ser facilmente explicada pelas diferenças observadas na morfologia pulmonar a partir das TCS. Como os pacientes sem ponto de inflexão inferior tinham lobos superiores bem ventilados e lobos inferiores não ventilados em ZEEP, parece lógico que a complacência pulmonar dos lobos superiores fosse muito maior do que a dos lobos inferiores. Quando níveis maiores de PEEP foram aplicados no sistema respiratório como um todo, houve aumento de volume das áreas pulmonares previamente ventiladas além de recrutamento das áreas não ventiladas, induzindo mais predominantemente hiperdistensão do que recrutamento alveolar.

Em conclusão, o uso da TC na IRA permite avaliar a morfologia pulmonar, medir a quantidade de gás e tecido no interior dos pulmões, calcular os volumes pulmonares totais e das diferentes regiões de interesse (ventilada, parcialmente ventilada, não ventilada e de hiperdistensão). A partir destes cálculos é possível quantificar o recrutamento alveolar e a hiperdistensão decorrentes de modalidades ventilatórias, como por exemplo do uso de diferentes níveis de PEEP.

\section{REFERÊNCIAS}

1. Rommelschein K, Lakner K, Westhofen P, Distelmaier W, Hirt S. Das respiratorische distress-syndrome der erwachensen (ARDS) om computer tomogram. A naesth Intensiether Notfallmed 1983;18:59-64.

2. Maunder RJ, Shuman WP, MCHugh JW, Marglin SI, Butler J. Preservation of normal lung regions in the adult respiratory distress syndrome. JAMA 1986;255:2463-2465.

3. Gattinoni L, Mascheroni D, Torresin A, Marcolin R, Fumagalli R, Vesconi S, Rossi G, Rossi F, Baglioni S, Bassi F, Nastri F, Pesenti A. Morphological response to positive end expiratory pressure in acute respiratory failure. Computerized tomography study. Intensive Care Med 1986;12:137-142.

4. Gattinoni L, Pesenti A, Torresin A, Baglioni S, Rivolta M, Rossi F, Scarani F, Marcolin R, Cappelletti G. Adult respiratory distress syndrome profiles by computed tomography. J Thorac Imag 1986;1:2530.

5. Gattinoni L, Pesenti A, Avalli L, Rossi F, Bombino M. Pressure-volume curve of total respiratory system in acute respiratory failure. Computed tomographic scan study. Am Rev Resp Dis 1987;136:730-736.

6. Gattinoni L, Pesenti A, Bombino M, Baglioni S, Rivolta M, Rossi G, Rossi G, Marcolin R, Mascheroni D, Torresin A. Relationships between lung computer tomographic density, gas exchange, and PEEP in acute respiratory failure. Anesthesiology 1988;69:824-832.

7. Gattinoni L, Pesenti A, Baglioni S, Vitale G, Rivolta M, Pelosi P. Inflammatory pulmonary edema and positive end-expiratory pressure : correlations between imaging and physiologic studies. J Thorac Imag 1988;3:59-64.

8. Gattinoni L, Pelosi P, Pesenti A, Bazzi L, Vitale G, Moretto A, Crespi A, Tagliabue M. CT scan in ARDS; clinical and physiopathological insights. Acta Anaesthesiol Scand 1991;35(Suppl 95):87-96.

9. Gattinoni L, Pelosi P, Vitale G, Pesenti A, D'Andrea L, Mascheroni D. Body position changes redistribute lung computed tomographic density in patients with acute respiratory failure. Anesthesiology 1991; 74:15-23.

10. Gattinoni L, Pelosi P, Crotti S, Valenza F. Effects of positive end-expiratory pressure on regional distribution of tidal volume and recruitment in adult respiratory distress syndrome. Am J Resp C rit Care Med 1995;151:1807-1814.

11. Pelosi P, Crotti S, Brazzi L, Gattinoni L. Computed tomography in adult respiratory distress syndrome: what has it taught us? Eur Respir J 1996;9:1055-1062.

12. U mamaheswara Rao GS, Gallart L, Law-koune J-D, Lu Q, Puybasset $L$, Coriat $P$, Rouby JJ. Factors influencing the uptake of inhaled nitric oxide in patients on mechanical ventilation. Anesthesiology 1997; 87:823-834.

13. Kollef $M H$, Schuster DP. The acute respiratory distress syndrome. $\mathrm{N}$ Engl J Med 1995;332:27-37. 
14. Gattinoni L, D'Andrea L, Pelosi P, Vitale G, Pesenti A, Fumagalli R. Regional effects and mechanism of positive end-expiratory pressure in early adult respiratory distress syndrome. J AMA 1993;269:2122-2127.

15. Pelosi P, D'Andrea L, Pesenti A, Gattinoni L. Vertical gradient of regional lung inflation in adult respiratory distress syndrome. Am J Resp Crit Care Med 1994;149:8-13.

16. Hedlund LW, Effmann EL, Bates WM, Beck J W, Goulding PL, Putman CE. Pulmonary edema: a CT study of regional changes in lung density following oleic acid injury. J Comput Assist Tomogr 1982;6:939-946.

17. Slutsky RA, Long S, Peck WW, Higgins CB, Mattrey R. Pulmonary density distribution in experimental noncardiac canine pulmonary edema evaluated by computed transmission tomography. Invest Radiol 1984;19:168-173.

18. Hylkema BS, Barkmeijer-Degenhart $P$, Van der Mark TW, Peset $R$, Sluiter $\mathrm{HJ}$. Measurement of functional residual capacity during mechanical ventilation for acute respiratory failure. Chest 1982;81:2730 .

19. Ibanez J, Raurich J M, Moris SG. Measurement of functional residual capacity during mechanical ventilation. Comparison of a computerized open nitrogen washout method with a closed helium dilution method. Intensive Care Med 1983;9:91-93.

20. East TD, Wortelboer PJ M, Van Ark E, Bloem FH, Peng L, Pace NL, Crapo RO, Drews D, Clemmer TP. Automated sulfur hexafluoride washout functional residual capacity measurement system for any mode of mechanical ventilation as well as spontaneous respiration. C rit Care Med 1990;18:84-91

21. Macnaughton PD, Evans TW. Measurement of lung volume and DLCO in acute respiratory failure. A m J Resp Crit Care Med 1994;150:770775

22. Dambrosio M, Roupie E, Mollet JJ, Angalde MC, Vasile N, Lemaire F, Brochard L. Effects of positive end-expiratory pressure and different tidal volumes on alveolar recruitment and hyperinflation. A nesthesiology 1997;87:495-503.

23. Vieira SRR, Puybasset L, Richecoeur J, Lu Q, Cluzel P, Gusman PB, Coriat $P$, Rouby J J. A lung computed tomographic assessment of positive end-expiratory pressure-induced lung overdistension. Am J Resp Crit Care Med 1998;158:1571-1577.

24. Vieira SRR, Putbasset L, Lu Q, Richecoeur J , Cluzel P, Coriat P, Rouby J - J. A scannographic assessment of pulmonary morphology in acute lung injury: signification of the lower inflection point detected on the lung pressure-volume curve. Am J Resp Crit Care Med 1999; (in press).

25. Levi C, Gray JE, McCullough EC, Rattery RR. The unreliability of CT numbers as absolute values. Am J Roentgenol 1982; 139:443-447.

26. Mergoni M, Martelli A, Volpi A, Primavera S, Zuccoli P, Rossi A. Impact of positive end-expiratory pressure on chest wall and lung pressure-volume curve in acute respiratory failure. Am J Resp Crit Care Med 1997;156:846-854. 